\title{
Desenvolvimento de um sistema para o controle do pH da água para microirrigação
}

\author{
Marinaldo F. Pinto ${ }^{1}$, Antonio P. Camargo ${ }^{1}$, Alexsandro C. S. Almeida ${ }^{1}$, \\ Dinara G. Alves ${ }^{1} \&$ Tarlei A. Botrel ${ }^{1}$
}

\begin{abstract}
RESU MO
O objetivo deste trabalho foi desenvolver um sistema eletrônico de controle do $\mathrm{pH}$ da água para microirrigação. $O$ trabalho foi realizado no Laboratório de Hidráulica do Departamento de Engenharia de Biossistemas (LEB) da Escola Superior de Agricultura "Luiz de Queiroz" (ESALQ/USP). O sistema de controle consta de um sensor de $\mathrm{pH}$, um circuito eletrônico programado para manter o pH da água em 5,5 e um dispositivo de injeção de ácido controlado por uma válvula solenóide comandada por pulsos elétricos enviados pelo circuito eletrônico. 0 sistema foi testado em águas de diferentes valores iniciais de $\mathrm{pH}$ (variando de 6,2 a 9,2) sob vazão constante $\left(264 \mathrm{~L} \mathrm{~h}^{-1}\right)$ e variável, sendo efetuada a medição do $\mathrm{pH}$ imediatamente à jusante do sistema e ao longo da linha lateral, nos pontos situados a 1, 14, 29 e 44 $\mathrm{m}$ do início e em diferentes intervalos de tempo a partir do início dos ensaios. O s resultados obtidos comprovam a eficiência do sistema para manter o pH da água na faixa desejada, tanto para a condição de vazão constante quanto para a variável. Não foram verificadas variações temporais nem espaciais significativas na linha lateral de irrigação.
\end{abstract}

Palavras-chave: controle de obstrução, precipitação de íons, tratamento de água

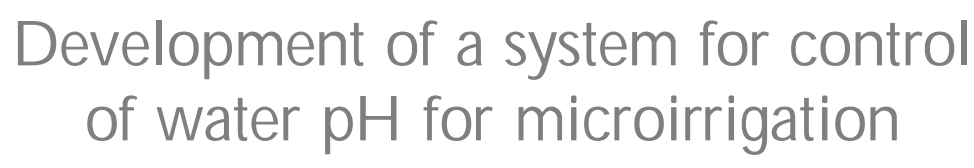

\begin{abstract}
The aim of this work was to develop an electronic system for control of water $\mathrm{pH}$ in microirrigation. The study was accomplished in the Hydraulics Laboratory of Biossystems Engineering Department, Escola Superior de Agricultura "Luiz de Q ueiroz" (ESALQ/U SP). The water pH control system has a device for acid injection controlled by a solenoid valve. This valve is switched on by electric pulses from a microcontroller. The system was evaluated for different water $\mathrm{pH}$ values (ranging from 6.2 to 9.2) under constant ( $\left.264 \mathrm{~L} \mathrm{~h}^{-1}\right)$ and variable flow. The $\mathrm{pH}$ was measured immediately on system downstream, and al ong the lateral line at 1, 14, 29, and $44 \mathrm{~m}$ from the lateral inlet, tests were also accomplished for different intervals of time. The obtained results proved the efficiency of the system on maintaining $w$ ater $\mathrm{pH}$ in desired range for constant and variable flow conditions. M eaningful temporal or spatial variations along lateral irrigation line were not observed.
\end{abstract}

Key words: clogging control, ions precipitation, treatment water 


\section{INTRODUÇÃO}

A escassez de água tem proporcionado uma procura maior por métodos de irrigação que possibilitem um aumento na eficiência do uso da água (Ribeiro \& Paterniani, 2007; Ribeiro et al., 2005a; Airoldi et al., 2003). Sendo assim, a microirrigação se apresenta como um método capaz de proporcionar, além de melhor eficiência, uma série de vantagens, como facilidade e eficiência na aplicação de fertilizantes, menor exigência de mãode-obra e redução dos riscos de contaminação de alimentos por organismos patogênicos; no entanto, este método faz uso de emissores de pequeno diâmetro que, por sua vez, apresentam alta susceptibilidade ao entupimento (Ravina et al., 1992).

Com isto, a qualidade da água pode restringir a adoção de sistemas de microirrigação (Scatolini \& Paterniani, 2001). O processo de obstrução de emissores pode ocorrer devido a partículas sólidas presentes na água de natureza física, química e/ou biológica, reduzindo a uniformidade de aplicação de água e a eficiência da irrigação (Ribeiro et al., 2008). Portanto, a qualidade da água utilizada na microirrigação é de fundamental importância para o desempenho desse sistema.

Deve-se levar em consideração, na escolha e no dimensionamento do sistema de filtragem, a natureza dos problemas da água de irrigação (Oliveira et al., 2006). A maioria dos problemas de obstrução em sistemas de microirrigação se deve a causas de natureza física (Adin \& Alon, 1986), de modo que o sistema de filtragem para essas circunstâncias deve impedir que as partículas sólidas em suspensão atinjam os emissores. Sistemas de filtragem para prevenir problemas de entupimento desta natureza, geralmente são constituídos pela associação de filtros de areia, de tela e/ou de discos, sendo que o primeiro é recomendado para retenção de contaminantes orgânicos e de algas (Testezlaf, 2008), e os outros para partículas sólidas de pequenos diâmetros.

Problemas de origem biológica são caracterizados pela obstrução de emissores devido à proliferação de microorganismos na água de irrigação e nas tubulações (Şahin et al., 2005), sendo que o cloro é a principal substância utilizada no controle de obstrução por razões desta natureza.

$\mathrm{Na}$ forma solúvel, os elementos ferro, manganês, cálcio e enxofre, atravessam os filtros sem serem retidos de modo que, ao reagirem com o oxigênio, precipitam e provocam a obstrução de emissores e/ou reduzem a seção de escoamento da tubulação, razão pela qual é oportuno um tratamento prévio da água de irrigação através da injeção de elementos químicos que venham intervir nas características da água, reduzindo a ocorrência de precipitação dos elementos citados e tornandoa apropriada à microirrigação. As principais substâncias químicas utilizadas com o objetivo de reduzir o potencial de obstrução devido a causas químicas são ácido clorídrico, ácido sulfúrico e ácido fosfórico.

O processo de precipitação é dependente do $\mathrm{pH}$, potencial redox, temperatura, pressão e concentração relativa de outras substâncias (Pitts et al., 2003), sendo que algumas dessas características não são passíveis de serem mensuradas em tempo real, como é o caso da concentração de bases. Considerando ainda que a qualidade da água pode sofrer mudanças temporais (Ribeiro et al., 2005a; 2005b), o controle de obstrução pode ser feito com base no $\mathrm{pH}$ da água, através da injeção de ácido, para manter o pH da água abaixo de 7 (Lamn et al., 2007; Pitts et al., 2003; Ayers \& Westcot, 1991), sendo que, quando este é mantido em níveis mais baixos, podese prevenir, também, a proliferação de micro-organismos, que também contribuem para a obstrução dos emissores.

Este trabalho teve como objetivo o desenvolvimento de um sistema de controle do $\mathrm{pH}$ da água em tempo real, diminuindo a possibilidade da ocorrência da precipitação de íons e a consequente obstrução dos emissores.

\section{Material e mÉtodos}

O trabalho foi desenvolvido no Laboratório de Hidráulica do Departamento de Engenharia de Biossistemas (LEB) da Escola Superior de Agricultura “Luiz de Queiroz" (Universidade de São Paulo - USP), onde o sistema eletrônico de controle do $\mathrm{pH}$ da água foi construído e realizados ensaios, visando verificar a capacidade do sistema em manter o $\mathrm{pH}$ da água em uma faixa previamente estabelecida. O controle do $\mathrm{pH}$ é feito com base na leitura do sensor acoplado ao sistema eletrônico controlador, que aciona uma válvula solenóide para a injeção de ácido na água. O tempo em que a válvula permanece aberta, é função do pH da água em cada instante de medição.

\section{Montagem do circuito eletrônico}

Os componentes eletrônicos utilizados na construção do circuito e seu arranjo, estão apresentados na Figura 1. O referido circuito pode ser descrito em quatro partes funcionais: a) amplificação do sinal de entrada; b) aquisição e processamento das informações (microcontrolador); c) controle dos atuadores

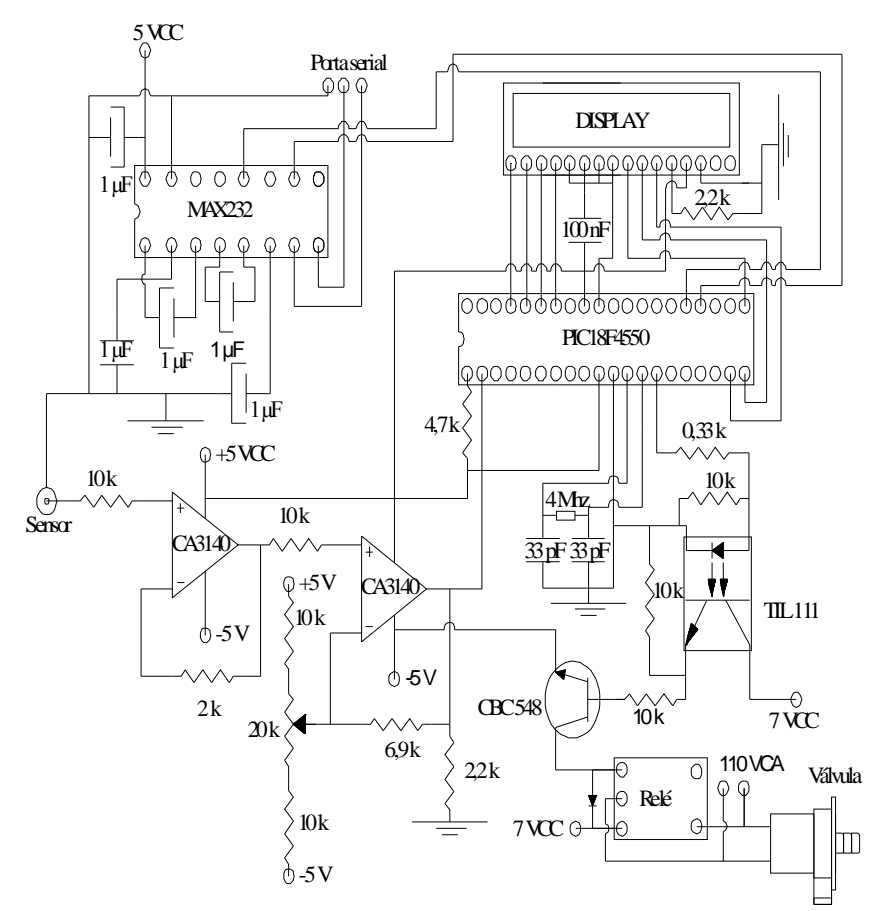

Figura 1. Esquema do circuito eletrônico utilizado na automação da injeção de ácido na água 
e d) interface para comunicação com o usuário. As características técnicas dos principais componentes estão apresentadas na Tabela 1 .

Tabela 1. Características técnicas dos principais componentes eletrônicos

\begin{tabular}{|c|c|c|}
\hline Componente & Característica & Unidade \\
\hline \multicolumn{3}{|c|}{ PIC18F4550 } \\
\hline Fabricante & Microchip Technology & - \\
\hline Alimentação & 5 & V \\
\hline$N^{\circ}$ pinos & 40 & - \\
\hline № de conversores $A D$ (10 bits) & 13 & - \\
\hline Portas & 5 & - \\
\hline Memória Flash & 32 & kBytes \\
\hline Memória EEPROM & 256 & Bytes \\
\hline Temperatura & -40 a 85 & ${ }^{\circ} \mathrm{C}$ \\
\hline Impedância de entrada máxima & 2,5 & $k \Omega$ \\
\hline \multicolumn{3}{|c|}{ MAX 232} \\
\hline Fabricante & Texas Instruments & - \\
\hline $\mathrm{N}^{0}$ pinos & 16 & - \\
\hline Temperatura & 0 a 70 & ${ }^{\circ} \mathrm{C}$ \\
\hline Alimentação & 5 & V \\
\hline \multicolumn{3}{|c|}{ CA 3140} \\
\hline Fabricante & Intersil & - \\
\hline$N^{\circ}$ pinos & 8 & - \\
\hline Temperatura & -25 a 150 & ${ }^{\circ} \mathrm{C}$ \\
\hline Alimentação & 4 a 36 & V \\
\hline Impedância de entrada & 1,5 & $\mathrm{~T} \Omega$ \\
\hline Impedância de saída & 60 & $\Omega$ \\
\hline
\end{tabular}

O circuito foi montado em uma placa PCI universal 10x10 cm; utilizou-se o microcontrolador PIC18F4550, da Microchip, que apresenta uma memória flash de 32 kbytes e é programado em linguagem $C$; para a transmissão de dados para computadores via interface serial, utilizou-se o circuito integrado MAX 232; além disso, o sistema é composto de display LCD para a visualização imediata do $\mathrm{pH}$ da água durante o processo de correção.

\section{MediçãodopH}

A medição do pH da água era realizada a cada segundo, através de um sensor potenciométrico, composto de um eletrodo de referência e um indicador, de modo que cada eletrodo constitui uma meia-célula. A meia-célula, que corresponde ao eletrodo indicador quando submetida a diferentes valores de $\mathrm{pH}$, gera uma diferença de potencial em relação ao eletrodo de referência, a qual é transmitida até o circuito medidor através de um cabo coaxial contendo um conector BNC universal.

A impedância dos sensores de pH é da ordem de $100 \mathrm{M} \Omega$ (Maia \& Maia, 2005), de modo que não é possível se fazer a leitura da diferença de potencial entre os eletrodos diretamente em um multímetro, ou no conversor analógico-digital, pois esses possuem baixa impedância de entrada e, assim sendo, usou-se um circuito amplificador que apresenta alta impedância de entrada e baixa de saída, respectivamente, como pode ser observado na Tabela 1.

Além do problema da impedância, quando o sensor é submetido a uma solução com $\mathrm{pH}$ maior que 7, gera-se uma diferença de potencial negativa, ao passo que em $\mathrm{pH}$ menor que 7 , o valor da diferença de potencial é positiva. O conversor analógico-digital (ADC) do microcontrolador tem como referência apenas valores de diferença de potencial positivos; portanto, não é possível se obter a leitura do sensor sem o ajuste do sinal, para $\mathrm{pH}$ maior que 7 , pois neste caso a diferença de potencial seria negativa; assim, o amplificador tem, como função, proporcionar um deslocamento (offset) na leitura do sensor, fazendo com que se tenha apenas valores positivos para qualquer valor de $\mathrm{pH}$.

A diferença de potencial gerada entre os eletrodos de indicação e de referência, além de ser função do pH da solução que se pretende mensurar é, também sensível a mudanças de temperatura, sendo que a $25^{\circ} \mathrm{C}$ seu valor é de $59,16 \mathrm{mV}$ para cada unidade de $\mathrm{pH}$; este valor varia linearmente de 54,20 a $66,10 \mathrm{mV}$, para uma temperatura de 0 e $60^{\circ} \mathrm{C}$, respectivamente (Figura 2). Deste modo, os medidores comerciais vêm acompanhados de um sensor de temperatura para compensação da variação da mesma. Neste trabalho tal fonte de erro foi desprezada, tendo em vista a pequena variação de temperatura da água nas condições avaliadas e a precisão necessitada pelo sistema.

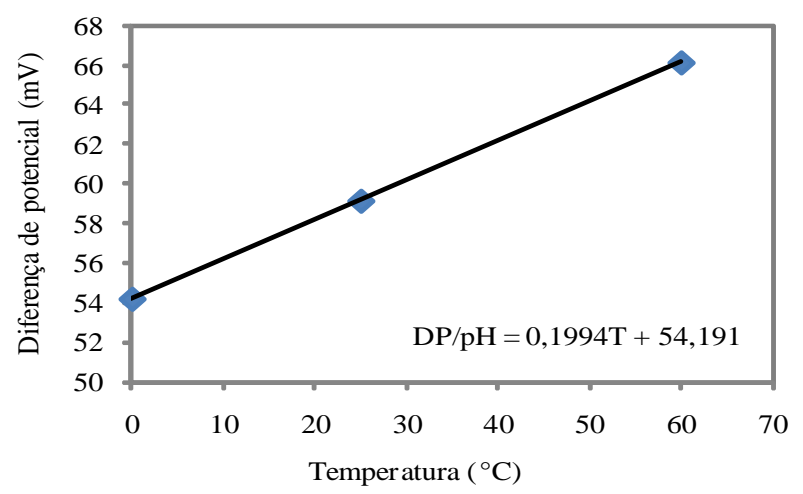

Figura 2. Resposta do sensor à variação de temperatura

Em função da alta impedância dos sensores de $\mathrm{pH}$, é comum a presença de ruídos nos sinais de resposta desses sensores; portanto, é de fundamental importância que os circuitos eletrônicos sejam dimensionados adequadamente e possuam sistema de aterramento eficiente (Maia \& Maia, 2005). No presente trabalho este problema foi contornado através da aquisição de um grande número de dados (1000) e se calculando sua média, procedimento que atua reduzindo erros aleatórios do processo de medição, minimizando o efeito de eventuais ruídos.

\section{Sistema de injeção de ácido}

O sistema é composto de uma válvula solenóide (Figura $3 \mathrm{~A})$ que injeta ácido fosfórico $\left(\mathrm{H}_{3} \mathrm{PO}_{4}\right)$ a $0,1 \mathrm{M}$ na tubulação, proveniente de um recipiente. O volume de ácido injetado é controlado pelo tempo que a válvula permanece aberta (TAV), sendo este tempo determinado pela lógica de controle proposta por Pinto (2010) e está restrito ao intervalo de 0 a $1 \mathrm{~s}$. Esta restrição se deve à taxa de aquisição de dados que é igual a $1 \mathrm{~s}$.

Para que o ácido seja injetado é imprescindível que sua pressão seja superior à da linha de irrigação e, deste modo, o ácido foi colocado em um recipiente a $1 \mathrm{~m}$ acima do nível do reservatório que abastece a linha lateral (Figura 3 B), 
A.

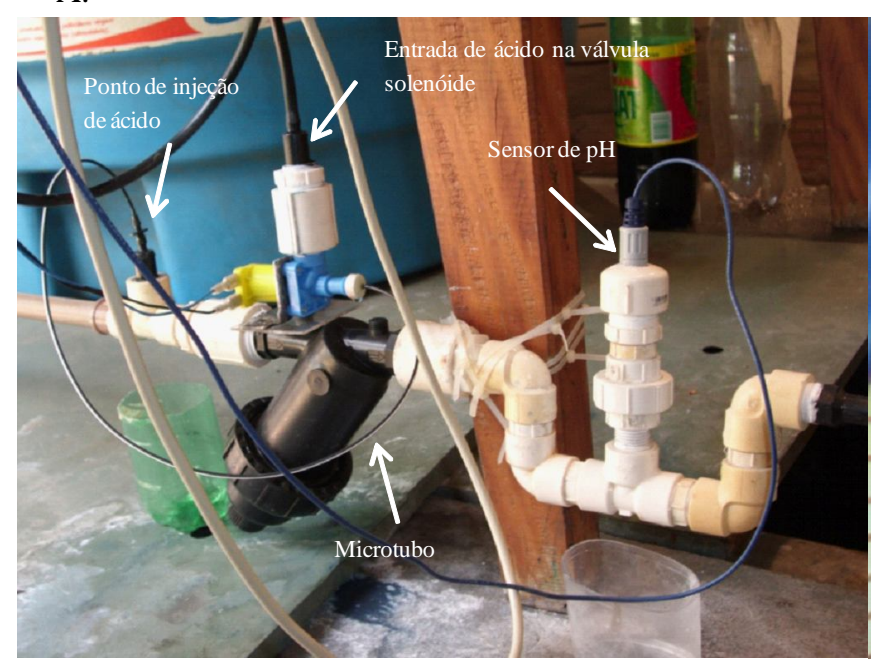

B.

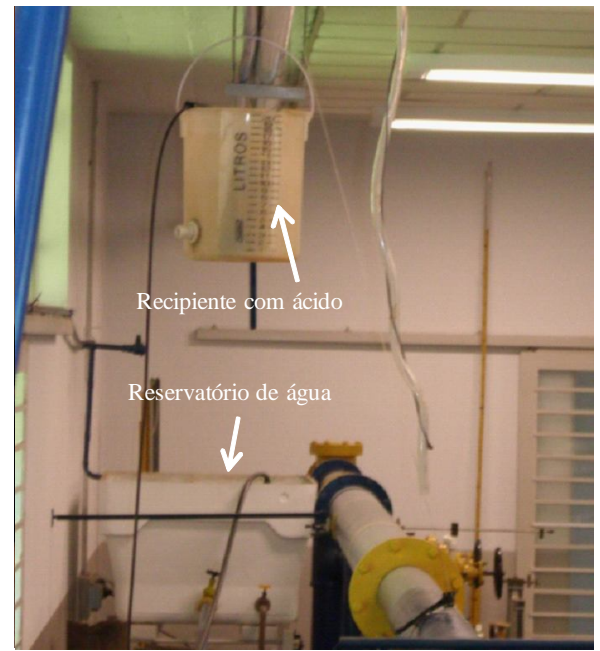

Figura 3. Sistema de controle do pH da água: (A) medição do $\mathrm{pH}$ e injeção de ácido; (B) recipiente com ácido

proporcionando a energia potencial necessária para que ocorra o fluxo de ácido desejado.

O sistema foi dimensionado para atender a uma demanda máxima de ácido, de modo a ajustar o pH de uma água com características químicas que a classifiquem como sendo de alto risco de obstrução dos emissores, proposto por Nakayama \& Bucks (1981), e para o limite superior dos valores normais de qualidade de água de irrigação, de acordo com Ayers \& Westcot (1991), sob uma vazão máxima de água igual a $280 \mathrm{Lh}^{-1}$. A vazão máxima de ácido necessária para a correção do $\mathrm{pH}$ da água foi calculada através do índice de Langelier (LSI).

Como a vazão de ácido em relação à de água é muito pequena, foi dimensionado um microtubo com diâmetro de $0,8 \mathrm{~mm}$, visando limitar a vazão de ácido na válvula solenóide, cujo dimensionamento foi feito para que o regime de escoamento seja laminar, conforme a metodologia apresentada por Almeida et al. (2009).

\section{Avaliação do sistema}

Verificou-se a eficiência do sistema no ajuste do $\mathrm{pH}$ da água, através da realização de ensaios do controlador, para águas com diferentes valores de $\mathrm{pH}$ inicial para vazão constante e sob a condição de vazão variável.

O sistema de controle foi acoplado a uma tubulação de 25 $\mathrm{mm}$, responsável pela condução de água para a uma linha lateral composta de microtubos, dimensionada para uma vazão de $264 \mathrm{~L} \mathrm{~h}^{-1}$. A linha estava situada em um terreno com declividade de $2,3 \%$ e apresentava comprimento de $44 \mathrm{~m}$, totalizando 44 microtubos espaçados $1 \mathrm{~m}$ entre si, sendo que cada microtubo emissor foi dimensionado para proporcionar uma vazão de $6 \mathrm{~L}$ $\mathrm{h}^{-1}$.

Para verificação do $\mathrm{pH}$ da água ao longo da linha lateral, foram feitas coletas de água em pontos situados a 1, 14, 29 e 44 m que correspondem aproximadamente ao início, 1/3, 2/3 e final da linha lateral, em diferentes tempos a partir do início do ensaio $(0,30,60,90$ e $120 \mathrm{~min})$, em que o pH das amostras de água foi mensurado imediatamente após cada coleta.

\section{RESULTADOS E DISCUSSÃO}

\section{Resposta do sensor à mudança de pH}

A Figura $4 \mathrm{~A}$ apresenta a resposta do sensor à variação de $\mathrm{pH}$ a $23{ }^{\circ} \mathrm{C}$, medida em um multímetro digital, na saída de um amplificador operacional, uma vez que não é possível medir diretamente a diferença de potencial em sensores de $\mathrm{pH}$, em razão da alta impedância desses sensores.

Observa-se que a diferença de potencial nos terminais do sensor foi de $56,289 \mathrm{mV}$ por unidade de $\mathrm{pH}$, sendo que os valores de diferencial de potencial medidos foram de 168,7, -3,7 e $-113,3 \mathrm{mV}$ para as soluções padrão de $\mathrm{pH} \mathrm{4,7}$ e 9, respectivamente, resultados que diferem da especificação do fabricante, que apresenta a resposta do sensor em função da temperatura de medição da solução. A diferença de potencial por unidade de $\mathrm{pH}$ (diferença de potencial unitária), apresentada pelo fabricante foi de 54,20 e $66,10 \mathrm{mV}$, para uma temperatura de 0 e $60{ }^{\circ} \mathrm{C}$, respectivamente, sendo que para valores de temperatura intermediários a resposta do sensor tem comportamento linear; logo, para a temperatura de $23{ }^{\circ} \mathrm{C}$, o valor de diferença de potencial elétrico seria de $58,76 \mathrm{mV}$; além do mais, o fabricante afirma que a resposta do sensor em $\mathrm{pH} 7$ é de $0 \mathrm{mV}$, porém se obteve valor de $-3,7 \mathrm{mV}$, diferença esta chamada de potencial de assimetria e é variável ao longo do tempo, o que justifica a calibração periódica de sensores.

Conforme a especificação do fabricante, o sensor apresenta resposta linear à variação de $\mathrm{pH}$, sendo que o modelo ajustado através de regressão linear apresentou coeficiente de determinação $\left(\mathrm{R}^{2}\right)$ de 0,9997 para o ensaio realizado (Figura 4 A), ou seja, praticamente $100 \%$ da variância são explicados pelo modelo de regressão.

A influência da temperatura na resposta do sensor foi analisada para uma variação de temperatura de 15 a $30^{\circ} \mathrm{C}$ tendo, como referência, a diferença de potencial a $25^{\circ} \mathrm{C}$, em uma faixa de -150 a $150 \mathrm{mV}$ ( $\mathrm{pH}$ de 9,5 a 4,5), o que abrange toda a faixa de valores de $\mathrm{pH}$ das águas de irrigação. A variação máxima no valor do $\mathrm{pH}$ foi de $0,93 \%$, inferior à precisão necessitada para esta aplicação; logo, nas condições avaliadas o compensador de temperatura é dispensável. 
A.

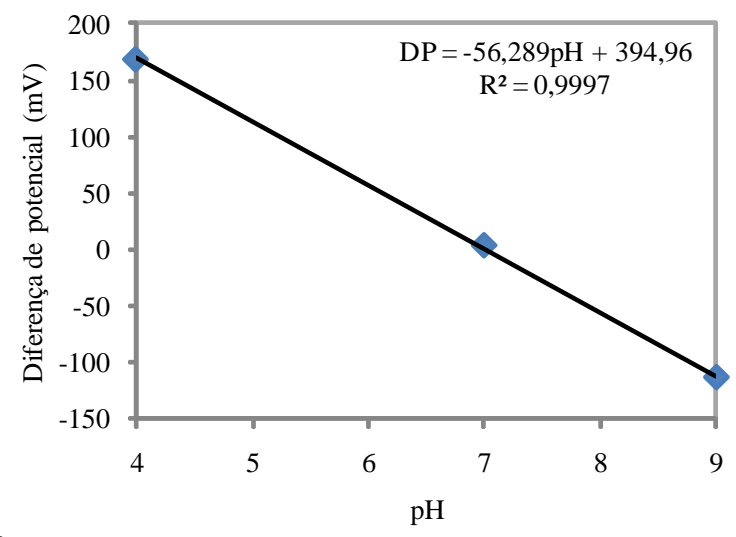

B.

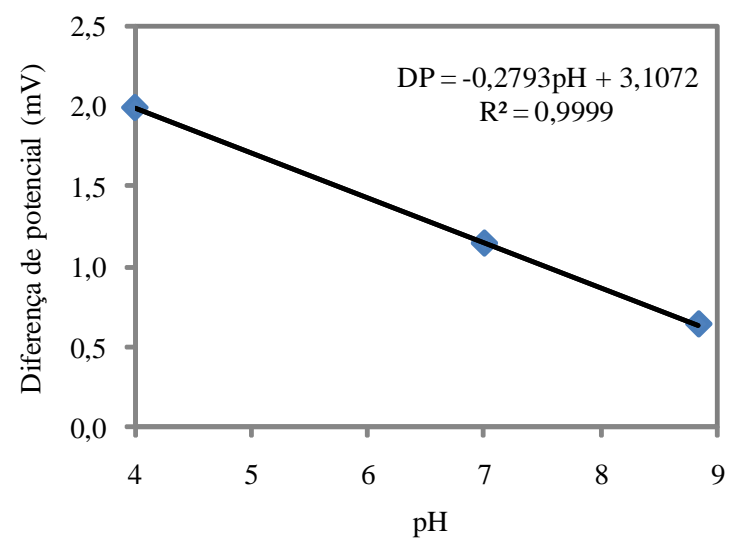

C.

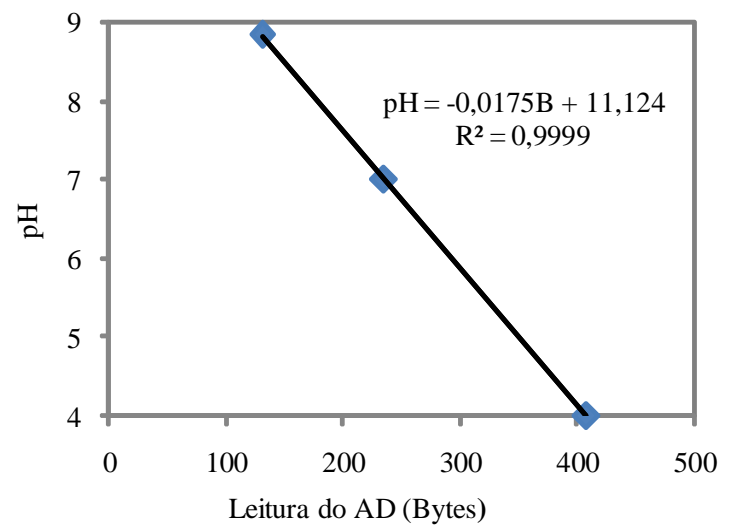

D.

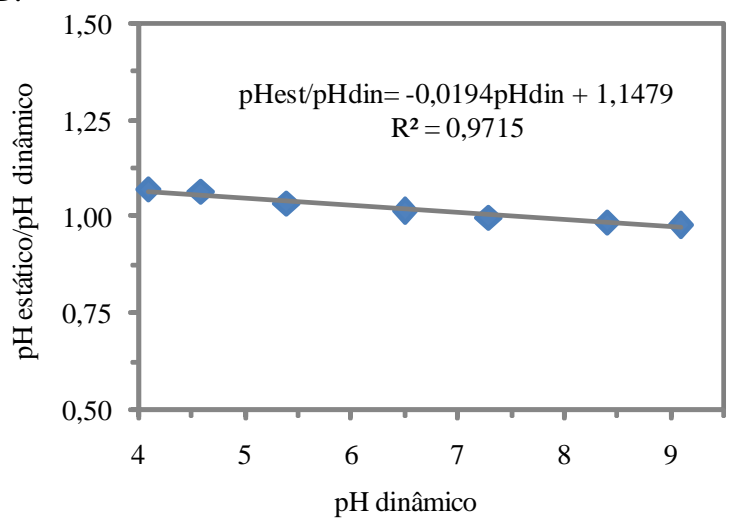

Figura 4. Resposta do sensor de $\mathrm{pH}$ : (A) sem amplificação de sinal elétrico $\left(23^{\circ} \mathrm{C}\right)$; (B) Sinal elétrico amplificado e com offset; (C) Leitura do ADC; (D) Correção da medida do $\mathrm{pH}$ sob a condição de água em movimento
Amplificação do sinal de entrada e ajuste do sinal de saída (offset)

O ADC do microcontrolador utilizado no circuito de controle, funciona em uma faixa de 0 a $5 \mathrm{~V}$, com resolução máxima de 10 bits; sendo assim, o sinal de entrada foi amplificado para melhorar a precisão do sistema; além disso, ajustou-se o sinal de saída do circuito amplificador para que a diferença de potencial fosse sempre positiva uma vez que apresenta valores negativos como pode ser observado na Figura 4A.

A curva de resposta do sensor depois da amplificação e ajuste do sinal elétrico, através de offset, está apresentada na Figura 4B, tendo como coeficiente de determinação 0,9999. Observa-se que o ajuste de offset proporcionou uma diferença de potencial de $1,146 \mathrm{~V}$ para um $\mathrm{pH} 7$, que representa a metade da escala de $\mathrm{pH}$, sendo que o valor correspondente na escala do conversor analógico-digital é de 2,5 V, porém, quando submetido ao offset, o sistema de amplificação atingiu a saturação para um sinal elétrico de $2,9 \mathrm{~V}$, restringindo a faixa de medição, visando a um ganho para o qual o sistema foi dimensionado (ganho igual a 5 vezes).

O ganho real fornecido pelo amplificador foi de 4,91 vezes, diferindo do valor previsto, que foi de 5 vezes; isto se deve à variação das características elétricas dos componentes que compõem o circuito amplificador e daí a necessidade da sua aferição.

Como o ajuste de offset do amplificador é feito manualmente, através de um resistor variável (trimpot) que pode sofrer variações com o tempo alterando a resposta do sensor, a calibração periódica do mesmo é essencial para a confiabilidade das medidas e consequente controle do $\mathrm{pH}$ da água.

A equação de calibração (Figura 4C) obtida através de regressão linear e utilizada na rotina de programação do microcontrolador, apresenta restrição quanto à escala de $\mathrm{pH}(0$ a 14). Pode-se constatar, através da equação de regressão, que o pH máximo de medição do sistema é de 11,124, quando o conversor é submetido a uma diferença de potencial de $0 \mathrm{~V}$; para valores de $\mathrm{pH}$ superiores a 11,124, o conversor estará sob diferença de potencial negativo e então a leitura do ADC é tomada como valor nulo.

O limite inferior do sistema consiste no $\mathrm{pH}$, que proporciona uma diferença de potencial de 2,9 V (potencial de saturação do amplificador operacional) no ADC; este valor é de 0,669 , de acordo com a equação de regressão da Figura 4C.

A solução para abranger toda a faixa de medição de $\mathrm{pH}(0$ a 14), seria reduzir o ganho do amplificador porém a faixa de $\mathrm{pH}$ das águas de irrigação é mais estreita, razão por que se optou por mantê-lo nesta condição.

\section{Resposta do sensor na condição de escoamento}

Quando o sensor de pH sofre perturbação mecânica, suas características se alteram; portanto, a medição do pH em condições de água em movimento difere daquele medido sob a condição estática, conforme se verifica na Figura 4D, que relaciona o pH da água medido sob condição estática e dinâmica, constatando-se que esta diferença apresentou comportamento linear com a razão entre o pH estático e o dinâmico. 
Observa-se, na Figura 4 D, que os dados estão um pouco dispersos da reta de regressão, o que proporciona um valor de coeficiente de determinação de 0,9715 , em virtude da precisão do aparelho medidor, que era de 0,1 , ou seja, os valores de $\mathrm{pH}$ intermediários não foram contabilizados no processo de medição; mesmo assim, o ajuste da função é satisfatório.

A influência do movimento da água na medida do $\mathrm{pH}$ depende da faixa em que ele é mensurado, de modo que, quando o pH se encontra superior a 7, a medida é subestimada, enquanto em pH menor que 7 este valor é superestimado; já para um pH próximo de 7 os valores praticamente coincidem.

\section{Ajuste do pH da água sob vazão constante}

O sistema mostrou-se eficiente para diferentes valores iniciais de $\mathrm{pH}$, compreendendo uma faixa de 6,2 até 9,2 (Figura $5 \mathrm{~A})$, sob vazão constante. O tempo de resposta do controlador para estabilização do $\mathrm{pH}$ em torno do valor desejado $(5,5)$, ocorreu por volta de 1 minuto para todos os valores iniciais de pH da água ensaiados, o que comprova a eficiência do sistema de controle, primeiro para estimar a vazão de ácido necessário para manter o $\mathrm{pH}$ da água ao valor estabelecido e depois em mantê-lo neste valor, de forma estável.

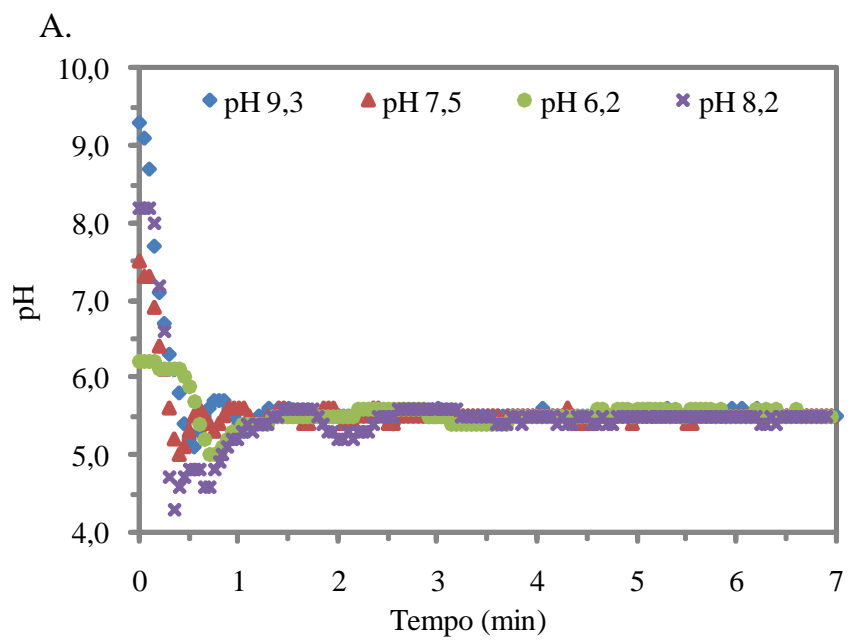

B.

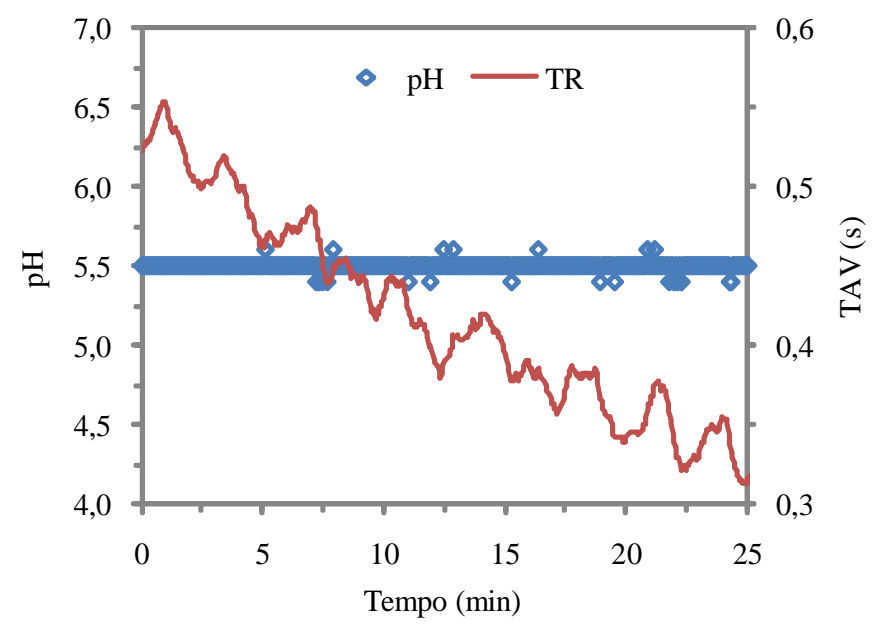

Figura 5. Ajuste do $\mathrm{pH}$ da água para diferentes valores iniciais, em condição de vazão constante (A); Ajuste do pH da água e tempo de permanência da válvula aberta (TAV), em condição de vazão variável e decrescente (B)
O tempo de resposta do ajuste da vazão está muito mais relacionado com o hardware do que com o software, uma vez que a injeção de ácido é feita à montante do ponto de medição, atrasando a detecção da mudança de $\mathrm{pH}$, porém isto é necessário para que ocorra a mistura do ácido na água, tendo como dispositivo de mistura um filtro de disco; como a velocidade da água no filtro é baixa (em torno de $0,6 \mathrm{~m} \mathrm{~s}^{-1}$ ), ocorre defasagem de tempo entre a injeção de ácido e a medição do $\mathrm{pH}$ da água.

Apesar das oscilações observadas nos valores iniciais de $\mathrm{pH}$ depois que o sistema ajusta a vazão de ácido, o $\mathrm{pH}$ da água é mantido dentro de uma faixa de 5,4 a 5,6, próximo a 5,5 (Figura $5 \mathrm{~A}$ e B). Esta variação é perfeitamente permissível para a aplicação considerada uma vez que é desejável manter o pH da água na faixa de 4,5 a 5,0 (Kidder \& Hanlon, 2009), de 6,0 a 7,0 (Lamn et al., 2007), e abaixo de 7 (Pitts et al., 2003).

\section{Ajuste do pH da água sob vazão variável}

Os resultados dos testes realizados sob vazão variável estão apresentados na Figura 5 B, na qual se verifica a adequação da lógica de controle utilizada para a condição de instabilidade na demanda de ácido, sendo que tais perturbações podem ocorrer devido a mudanças no fluxo de água ou variações temporais de $\mathrm{pH}$ comumente observadas nas águas de rios e lagos (Ribeiro et al., 2005a). Nota-se que o sistema ajustou o pH da água de maneira similar à condição de vazão constante, ou seja, o pH da água se manteve entre 5,4 e 5,6, demonstrando que a lógica de controle utilizada foi adequada. $\mathrm{O}$ tempo em que a válvula de controle do volume de ácido injetado foi mantida aberta (TAV) foi decrescente no decorrer do ensaio devido à redução da vazão de água, o que reduz a necessidade de ácido para manter o $\mathrm{pH}$ da água dentro da faixa estabelecida, uma vez que a qualidade da água não sofreu variação durante a realização desses ensaios.

\section{Testes do sistema em uma linha lateral}

A Tabela 2 apresenta os resultados dos testes realizados na linha lateral podendo-se observar que não ocorreram diferenças significativas nos valores de $\mathrm{pH}$ nem nos valores de potencial de redução, a 5\% de significância; além disso, o pH médio em cada ponto medido se manteve nas proximidades do valor ajustado no início da linha lateral $(5,5)$, com variação máxima de $\pm 0,1$; a faixa de $\mathrm{pH}$ em que a precipitação de íons teoricamente não ocorreria, é mais ampla, podendo-se considerar satisfatórios os resultados obtidos.

Em relação ao tempo de funcionamento do sistema, constatou-se que tanto o pH quanto o potencial de oxirredução diferiram estatisticamente, e que o $\mathrm{pH}$ sofreu um decréscimo com o tempo, ocorrendo o inverso com o potencial redox.

A redução do $\mathrm{pH}$ está relacionada com a condição em que foi realizado o ensaio, pois o sistema era acionado com um pH inicial elevado, iniciando-se o processo de correção. Deste modo, a linha lateral de irrigação era abastecida, inicialmente, com uma água de $\mathrm{pH}$ mais elevado, refletindo no valor do $\mathrm{pH}$ medido ao longo da linha lateral, mesmo que o pH na saída do sistema de injeção estivesse corrigido para o valor estabelecido $(5,5)$. Em condições normais de irrigação este processo não ocorreria, uma vez que o sistema funcionaria sempre utilizando 
Tabela 2. Avaliação da variação do pH ao longo da linha lateral, após a correção da água (vazão de $264 \mathrm{~L} \mathrm{~h}^{-1}$ )

\begin{tabular}{cccccc}
\hline & \multicolumn{4}{c}{ Fração da linha lateral } & \multirow{2}{*}{ Média } \\
\cline { 2 - 4 } Tempo (min) & $\mathbf{0}$ & $\mathbf{1 / 3}$ & $\mathbf{2 / 3}$ & $\mathbf{1}$ & \\
\cline { 2 - 4 } & \multicolumn{4}{c}{$\mathbf{p H}$} \\
0 & 5,68 & 5,64 & 5,78 & 5,64 & $5,69 \mathrm{~A}$ \\
30 & 5,76 & 5,61 & 5,68 & 5,65 & $5,68 \mathrm{~A}$ \\
60 & 5,59 & 5,50 & 5,55 & 5,53 & $5,54 \mathrm{AB}$ \\
90 & 5,62 & 5,44 & 5,54 & 5,49 & $5,52 \mathrm{~B}$ \\
120 & 5,63 & 5,52 & 5,55 & 5,57 & $5,57 \mathrm{~B}$ \\
\hline Média & $5,66 \mathrm{~A}$ & $5,54 \mathrm{~A}$ & $5,62 \mathrm{~A}$ & $5,58 \mathrm{~A}$ \\
\hline Tempo (min) & \multicolumn{3}{c}{ Potencial de oxirredução (mV) } \\
0 & 546,7 & 581,6 & 580,2 & 561,1 & $567,4 \mathrm{~A}$ \\
30 & 619,5 & 629,3 & 622,4 & 601,8 & $618,3 \mathrm{~B}$ \\
60 & 682,0 & 670,8 & 655,7 & 662,9 & $667,9 \mathrm{BC}$ \\
90 & 681,2 & 675,5 & 666,0 & 686,2 & $677,2 \mathrm{C}$ \\
120 & 694,4 & 692,9 & 699,9 & 703,7 & $697,7 \mathrm{C}$ \\
\hline Média & $644,8 \mathrm{~A}$ & $650,0 \mathrm{~A}$ & $644,8 \mathrm{~A}$ & $643,1 \mathrm{~A}$ \\
\hline As médias seguidas de mesma letra não diferem entre si, pelo teste de Tukey a 5 \% de significância
\end{tabular}

água com pH corrigido, contribuindo para um pH mais estável na saída dos emissores.

O aumento do potencial de redução é, portanto, consequência da redução do $\mathrm{pH}$ da água, uma vez que esses parâmetros são inversamente proporcionais para a condição de equilíbrio, sendo este um dos motivos pelos quais se propõe o ajuste do $\mathrm{pH}$ da água para o controle de obstrução de emissores, em microirrigação.

\section{CONCLUSÕES}

1. O circuito de leitura de $\mathrm{pH}$ mostrou-se eficiente na obtenção deste parâmetro para a aplicação.

2. A técnica de controle utilizada foi eficaz no ajuste do $\mathrm{pH}$ da água.

3. A medida do $\mathrm{pH}$ da água na condição dinâmica difere daquela feita sob a condição estática, sendo necessária sua correção.

4. O sistema desenvolvido pode ser empregado no controle do $\mathrm{pH}$ da água para a microirrigação.

\section{LITERATURA CITADA}

Adin, A.; Alon, G. Mechanisms and process parameters of filter screens. Journal of Irrigation and Drainage Engineering, v.112, n.4, p.293-304, 1986.

Airoldi, R. P. S.; Paterniani, J. E. S.; Ribeiro, T. A. P.; Silva, M. J. M. Comparação entre elementos filtrantes de manta sintética não tecida e de disco no tratamento de água para irrigação. Irriga, v.8, n.3, p.218-233, 2003.

Almeida, D. C. G. C.; Botrel, T. A.; Smith, R. J. Characterization of the microtubes emitters used in novel micro-sprinkler. Irrigation Science, v.27, n.3, p.209-214, 2009.
Ayers, R. S.; Westcot, D. W. A qualidade da água na agricultura. Campina Grande: UFPB, 1999.153p. Estudos FAO: Irrigação e Drenagem, 29

Kidder, G. E.; Hanlon, A. Neutralizing excess bicarbonates from Irrigation water. Florida: University of Florida, Florida Cooperative Extension Service, 2009. 12p.

Lamn, F. R.; Ayars, J.; Nakayama, F. S. Microirrigation for crop production: Design, operation, and management. Amsterdam: Elsevier Science Publishers, 2007.618p.

Maia, F. J. de O.; Maia, J. de O. Estudo da estabilidade e de outras características de um dispositivo eletrônico para a calibração de eletrodos de medição de pH. In: Encontro para a Qualidade de Laboratórios, 2005, São Paulo. Anais... São Paulo: REMESP, 2005. p.1-6.

Nakayama, F. S.; Bucks, D. A. Emitter clogging effects on trickle irrigation uniformity. Transaction of ASAE, v.24, p.77-80, 1981.

Oliveira, C. F.; Frizzone, J. A.; Rettore Neto, O. Perda de carga em filtros de tela e de discos utilizados na irrigação localizada. Engenharia Rural, v.17, p.21-28, 2006.

Pinto, M. F. Desenvolvimento de um sistema para o controle do pH da água para irrigação localizada. Piracicaba: ESALQ/ USP, 2010. 83p. Dissertação Mestrado

Pitts, D. J.; Haman, D. Z.; Smajstrla, A. G. Causes and prevention of emitter plugging in microirrigation systems. Florida: University of Florida, Florida Cooperative Extension Service, 2003. 12p. Bulletin 258

Ravina, I.; Paz, E.; Sofer, Z.; Marcu, A.; Shisha, A.; Sagi, G. Control of emitter clogging in drip irrigation with reclaimed wastewater. Irrigation Science, v.13, p.129-139, 1992.

Ribeiro, T. A. P.; Airoldi, R. P.S.; Paterniani, J. E. S.; Silva, M. J. M.. Variação temporal da qualidade da água no desempenho de filtros utilizados na irrigação por gotejamento. Revista Brasileira de Engenharia Agrícola e Ambiental, v.9, p.450456, 2005a.

Ribeiro, T. A. P.; Paterniani, J. E. S. Avaliação do efeito da cloração da água em sistemas de filtragem de irrigação localizada. Revista Brasileira de Engenharia de Biosistemas, v.1, p.31-39, 2007.

Ribeiro, T. A. P.; Paterniani, J. E. S.; Colleti, C. Chemical Treatment to Unclogg Dripper Irrigation Systems due to Biological Problems. Scientia Agricola, v.65, p.1-9, 2008.

Ribeiro, T. A. P.; Paterniani, J. E. S. ; Silva, M. J. M.; Airoldi, R. P. S. Efeito da qualidade da água na perda de carga em filtros utilizados na irrigação localizada. Revista Brasileira de Engenharia Agrícola e Ambiental, v.9, p.1-6, 2005b.

Şahin, Ü.; Anapalı, Ö.; Dönmez, M. F.; Şahin, F. Biological treatment of clogged emitters in a drip irrigation system. Journal of Environmental Management, v.76, p.338-341, 2005.

Scatolini, M. E.; Paterniani, J. E. S. Remoção de sólidos suspensos na água de irrigação utilizando mantas sintéticas não tecidas. Revista Brasileira de Engenharia Agrícola e Ambiental, v.5, n.3, p.382-385, 2001.

Testezlaf, R. Filtros de areia aplicados à irrigação localizada: teoria e prática. Engenharia Agrícola, v.28, n.3, p.604-613, 2008. 